Pacific Journal of Mathematic 


\section{THE END POINT COMPACTIFICATION OF MANIFOLDS}

FRANK RAYMOND

Introduction. This paper originated in trying to show that the one point compactification of an orientable generalized $n$-manifold $(n$-gm) with cohomology isomorphic to Euclidean $n$-space was an orientable $n$-gm. Heretofore, in papers on transformation groups where this was relevant, it was stated as an extra assumption. ${ }^{1}$ The solution to this problem is given as a corollary to the main theorems which characterize the orientable (or locally orientable) generalized manifolds whose Freudenthal end point compactification is again an orientable (or locally orientable) generalized $n$-manifold (see 4.5 and 4.13). In the first section we give a new characterization of the Freudenthal end point compactification in terms of inverse limits. We show as in Specker [10] that a certain 0-dimensional cohomology group measures the extent of this compactification.

Higher dimensional analogues of this cohomology group have been used by Conner [4] in proving, e.g., that a simply connected locally Euclidean $n$-manifold whose 1 point compactification is a locally Euclidean $n$-manifold cannot be fibered by a non-trivial compact fiber. $\mathrm{He}$ has called these groups the cohomology of the ideal boundary. These groups are further studied and the homology analogue is derived (see $\S 2)$. The main Lemma (2.16) is an exact sequence which relates the homology of the ideal boundary with the homology of a given compactification and the local homology groups at infinity. This exact sequence together with our characterization of the Freudenthal compactification gives the main theorems.

Applications are given in $\S 3$ to Poincaré duality, in $\S 5$ to open 2manifolds, and in $\S 6$ special mappings of manifolds.

Throughout this paper $X$ will denote a locally campct, locally connected, connected Hausdorff space. If $S$ is a locally compact Hausdorff space, $A(S)$ will denote the collection of open subsets of $S$ whose closure is compact. When the generic space is not necessarily locally connected, as is usually the case in $\S 2$ and part of $\S 4$, it will be denoted by the letter $S$.

\section{The Freudenthal end point compacification.}

1.1. Lemma. If $V, U \in A(X)$ such that $\bar{V} \subset U$, then at most a finite number of components of $X-\bar{V}$ meet in $X-\bar{U}$. In particular,

Received July 14, 1959. National Science Foundation Fellow.

1 See, for example, Montgomery and Mostow, Toroid transformation groups on Euclidean space, I11. J. of Math. 2 (1958), or [14]. 
for every $V \in A(X)$ there is at most a finite number of unbounded ${ }^{2}$ components of $X-\bar{V}$.

1.2. Definition (Specker). The space $X$ has at least $k$ ends if there exists $V \in A(X)$ such that $X-\bar{V}$ has at least $k$ unbounded components. The space $X$ has exactly $k$ ends if $X$ has at least $k$ ends but not at least $k+1$ ends.

1.3. If $V, U \in A(X), \bar{V} \subset U$, then each unbounded component of $X-\bar{U}$ lies in an unbounded component $X-\bar{V}$. Furthermore, each unbounded component of $X-\bar{V}$ meets at least one unbounded component of $X-\bar{U}$. If $X$ has exactly $k$ ends then there exists $V \in A(X)$ so that $X-\bar{V}$ has exaclty $k$ unbounded components. Thus if $\bar{V} \subset U \in$ $A(X), U$ would have the same property.

Let us therefore in case $X$ has exactly $k$ ends, associate an ideal point with each unbounded component of $X-\bar{V}$. There is a one-to-one correspondence of the unbounded components of $X-\bar{U}$ with those of $X-\bar{V}$ as we have just seen. We may adjoion this ideal set of $k$ points to $X$ and specify that a neighborhond of such an ideal point will be the ideal point together with the unbounded component associated to the ideal point. Such a process yields a compactification of $X$ by $k$ points. It is exactly this process that we wish to extend in case $X$ has more than a finite number of ends.

1.4. Let $A$ index the set $A(X)$. Partially order $A$ by $\alpha<\beta$ if $V_{\alpha}, V_{\beta} \in A(X)$ and $\bar{V}_{\alpha} \subset V_{\beta}$. Let $A_{\alpha}=\left\{A_{\alpha}^{i}\right\}_{\alpha=1}^{i=\alpha(i)}$ denote the collection of unbounded components of $X-\bar{V}_{\alpha}$. If $\alpha<\beta$, let $\pi_{\alpha}^{\beta}: A_{\beta} \rightarrow A_{\alpha}$ be the mapping induced from the inclusion $\left(X-\bar{V}_{\beta}\right) \subset\left(X-\bar{V}_{\alpha}\right)$, i.e., $\pi_{\alpha}^{\beta}$ sends each unbounded component $A_{\beta}^{i}$ of $X-\bar{V}_{\beta}$ into the unique unbounded component of $X-\bar{V}_{\alpha}$ which contains $A_{\beta}^{i}$. Clearly, $\pi_{\alpha}^{\beta}$ is onto. The collection $\left\{A_{\alpha}, \pi_{\alpha}^{\beta}\right\}$ forms an inverse system of sets. Let $B=$ inverse limit $\left\{A_{\alpha}, \pi_{\alpha}^{\beta}\right\}$. Each set $A_{\alpha}$ is a finite set and if topologized by the discrete topology the space $B$ will be a compact space.

A topology will now be put on the set $X^{\prime}=X \cup B$. Let $a \in B$, and define a neighborhood system for the point $a$. Let $\alpha \in A$. Let $\pi_{\alpha}(\alpha)=A_{\alpha}^{\alpha(a)}$ be the undounded component of $A_{\alpha}$ which is the $\alpha$ th coordinate of $a$. Let $B^{\alpha(a)}$ be the set of points of $B$ so that $\pi_{\alpha}(b)=A_{\alpha}^{\alpha(a)}$. Then $N_{a}^{\alpha}=A_{\alpha}^{\alpha(a)} \cup B^{\alpha(a)}$ will be an element of the neighborhood system of $a \in B \subset X^{\prime}$. The neighborhood system of $a$ will be $\left\{N_{a}^{\alpha}\right\}_{\alpha \in A}$. If $x \in X \subset X^{\prime}$ a neighborhood system of $x$ will be given by just choosing a neighborhood system of $x$ in $X$.

1.5. Theorem. Let $X^{\prime}$ be the space obtained from $X$ as defined

2 A set is unbounded if its closure is not compact. 
above. Then,

(a) $X^{\prime}$ is Hausdorff, connected and locally connected,

(b) $B$ is closed and has no interior points in $X^{\prime}$,

(c) $B$ is totally disconnected in $X^{\prime}$,

(d) the topology of $B$ as an inverse limit is the relative topology of $B$ in $X^{\prime}$,

(e) $X^{\prime}$ is compact,

(f) if $O$ is an open connected set in $X^{\prime}$, then $O-B$ is connected.

Proof. The definition of $X^{\prime}$ together with the fact that every point of $B$ is a limit point of $X$ implies (a) and (b).

Let $a, b \in B, a \neq b$. Then there exists $\alpha \in A$ such that $\pi_{\alpha}(\alpha) \neq \pi_{\alpha}(b)$. Let $C=B-B^{\alpha(a)}$. Clearly $b \in C$ and $B^{\alpha(a)}$ is open in $B$ (not in $X^{\prime}$ ). Now, $\pi_{\alpha}(p)=\pi_{\alpha}(a)$ if $p$ is a limit point of $B^{\alpha(a)}$, hence $B^{\alpha(a)}$ is both open and closed. Consequently, $a$ and $b$ are separated in $B$, hence (c) holds.

Let $a \in B$ and $N_{a}^{\alpha}$ be an element of the neighborhood system of $a$. The set $B^{\alpha(a)}$ consists of all those points of $b \in B$ so that $\pi_{\alpha}(b)=A_{\alpha}^{\alpha(a)}=\pi_{\alpha}(a)$. However, in terms of the inverse limit topology on $B, B^{\alpha(a)}=\pi_{\alpha}^{-1}\left(A_{x}^{\alpha(a)}\right)$ is also an open set. Moreover, the neighborhoods of $a \in B$ in terms of the inverse limit topology on $B$ are generated precisely by the sets $B^{\alpha(a)}$. Hence, (d) holds and, therefore, $B$ is compact. It is now not hard to see that $X^{\prime}$ must be compact.

Let $O$ be any open connected set which meets $B$. The set $O$ may be regarded as the union of fundamental open connected sets of points of $O \cap B$. Each such neighborhood is connected and cannot be separated by removal of $B^{x(\alpha)}$, by definition of neighborhoods. An elementary argument now yields that $O-B$ must be connected. In particular, every point of $B$ is a local non-cut point of $X^{\prime}$.

1.6. Definition. The compactification $X^{\prime}$ of $X$ will be called the (Freudenthal) end point compactification of $X$.

1.7. The set $B$ contains exactly $k$ points, if and only if, $X$ has exactly $k$ ends. In case $k$ is finite, $X^{\prime}$ agrees with the preliminary definition of the end point compactification of 1.3 .

1.8. The consequences of Theorem 1.5 serve as an abstract characterization of the end point compactification.

THEOREM. Let $X^{*}$ be a compactification of $X$ such that:

(a) $X^{*}$ is connected;

(b) $X$ is open is $X^{*}$;

(c) $X^{*}-X$ is totally disconnected; 
(d) if $p \in X^{*}-X$ and $U$ is a connected neighborhood (open) of $p$ then $U-\left(X^{*}-X\right)$ is connected.

Then, $X^{*}$ may be identified with the end point compactification $X^{\prime}$ of $X$.

Proof. As $X^{*}$ is to be locally compact, it can fail to be locally connected only on a set containing a non-degenerate continuum [see e.g., 12, 2.2 , pp. 104]. But $X$ is locally connected and $B^{*}=X^{*}-X$ is totally disconnected, and therefore, $X^{*}$ must be locally connected. Moreover, $X^{*}-X$ has no interior points in $X^{*}$.

Let $p$ be a point of $B^{*}$. Let $U_{p}$ be a neighborhood such that $U_{p}-B^{*}$ is unbounded in $X$. Let $B^{*}-U_{p}=C$. Then as $B^{*}$ is totally disconnected $C$ and $p$ may be separated. Choose $C_{1}$ and $C_{2}$ such that $C_{1} \cup C_{2}=B^{*}, p \in C_{1}, C \subset C_{2}, C_{1} \cap C_{2}=\phi$. Since the $C_{i}$ are both closed and disjoint we can choose open disjoint sets $O_{1}$ and $O_{2}$ such that $O_{1} \supset C_{1}$, and $O_{2} \supset C_{2}$. We can choose both $O_{i}$ to be regular open sets (i.e., interior $\bar{O}_{i}=O_{i}$ ), and $O_{1} \subset U_{p}$. As $O_{1}$ and $O_{2}$ cover $B^{*}$ we may select from $O_{1}$ and $O_{2}$ just those components which meet $B^{*}$. Let $U=$ be the components of $O_{1}$ and $O_{2}$ which meet $B^{*}$. As $U$ is chosen to be a regular open set, $X^{*}-U=\bar{V}$ for some $V \in A(X)$. The collection $A^{\prime}(X)$ of all possible $V$ of this form is cofinal in $A(X)$. Now by taking the unbounded (in $X$ ) components of $X^{*}-V$ we can recover, by the process used in defining $X^{\prime}$, a cofinal neighborhood system of $p$. Thus it readily follows that $B^{*}$ is homeomorphic to the inverse limit of the inverse system of unbounded components of the complement of the closure of elements of $A^{\prime}(X)$. Thus $X^{*}$ may be identified with $X^{\prime}$ and $B^{*}$ with $B$.

1.9. The abstract characterization of the end point compactification used the same conditions as that of Freudenthal [7] although the postulates on the topology of $X$ are not quite the same.

1.10. In the case of a complex, Specker has measured the number of ends by the rank of a certain cohomology group, [10]. Analogously, the similar cohomology group, but now of the space $X$, gives the cohomology of $B$ (see 1.13).

Let $H^{*}(X)$ denote the Cech cohomology ring of $X$ with coefficients in the principal ideal domain $L$. Then following Conner [4] define $I^{0}(X)$ to be the direct limit of $H^{0}(X-V)=$ direct limit of $H^{0}(X-\bar{V})$, taken over the directed set $A, V \in A(X)$

The group $H^{0}(X-\bar{V})$ splits into the direct product of copies of $L$, one for each component. In the direct limit every contribution from an unbounded component is annihilated. In fact, if $X$ is paracompact it is easy to show that $I^{\circ}(X)$ is free and at most countably generated.

1.11. Let $X^{\prime}$ denote the end point compactificatoin of $X, X^{\prime}-X=$ 
$B$, and $V \in A(X)$. Consider the exact sequence:

1.12

$$
\begin{array}{r}
0 \longrightarrow H^{0}\left(X^{\prime}-V, X-V\right) \stackrel{j^{*}}{\longrightarrow} H^{0}\left(X^{\prime}-V\right) \stackrel{i^{*}}{\longrightarrow} H^{0}(X-V) \\
\stackrel{d^{*}}{\longrightarrow} H^{1}\left(X^{\prime}-V, X-V\right) \longrightarrow
\end{array}
$$

The group $H^{0}\left(X^{\prime}-V, X-V\right) \approx H^{0}\left(X^{\prime}, X\right)=0$, as $X^{\prime}$ is connected. The mapping $i^{*}: H^{0}\left(X^{\prime}-\bar{V}\right) \rightarrow H^{0}(X-\bar{V})$ is an isomorphism when restricted to the unbounded components (in $X$ ) of $X^{\prime}-\bar{V}$. Furthermore, the direct limit $H^{0}\left(X^{\prime}-\bar{V}\right)=\operatorname{direct}$ limit $H^{0}\left(X^{\prime}-V\right)$, and by continuity is isomorphic to $H^{0}(B)$. Passing the direct limit of 1.12 we obtain:

1.13. TheOREM. The mapping $i_{\infty}^{*}: H^{0}(B) \rightarrow I^{0}(X)$ is an isomorphism onto. Moreover, $H^{1}\left(X_{s} ; X^{\prime}-B\right)$ is 0 .

2. Cohomology and homology of the ideal boundary. In this section we shall recall the definition of end groups or the cohomology groups of the ideal boundary as given by Conner [4]. The higher dimensional analogue of (1.12) will be developed (2.6) and an analogous definition for homology of the ideal boundary will also be given. In $\S 3$ we turn to applications.

2.1. Let $C^{*}(\mathrm{~S})$ denote the grating of Alexander-Spanier cochains of the locally compact Hausdorff space $S$. The coefficient domain, usually suppressed, is taken to be a principal ideal domain, $L$. Let $C_{c}^{*}(S)$ denote the subgrating of elements of $C^{*}(S)$ which have compact support; $C_{c}^{*}(S)$ is an ideal in $C^{*}(S)$. The quotient ring is called the cohomology ring of the ideal boundary of $S$, or the end cohomology ring of $S$. It will be denoted, following Conner [4] by $I^{*}(S)=\sum_{p=0}^{\infty} I^{p}(S)$.

If $f$ is a proper mapping of $S$ into $T$ then the daigram

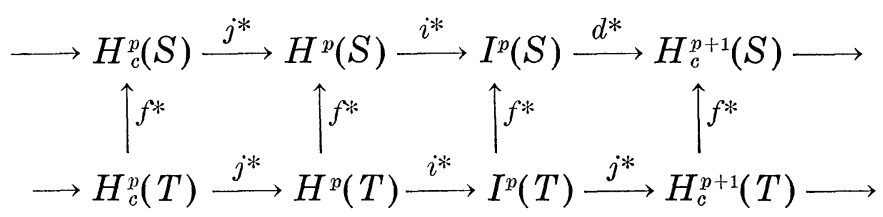

is commutative. That the horizontal rows are exact is a consequence of the exactness of the sequence of cochains:

$$
0 \longrightarrow C_{c}^{p}(S) \stackrel{j}{\longrightarrow} C^{p}(S) \stackrel{i}{\longrightarrow} C^{p}(S) / C_{c}^{p}(S) \longrightarrow 0 ;
$$

and the fact that $i$ and $j$ commute with the coboundary.

Let $U \in A(S)$, then it is to be remarked that (2.3) is the direct limit of:

$$
0 \longrightarrow C_{c}^{p}(U) \longrightarrow C^{p}(S) \longrightarrow C^{p}(S) / C_{c}^{p}(U) \longrightarrow 0
$$


The cohomology functor commutes with the direct limit and therefore the top horizontal line of (2.2) may be written as the direct limit of derived cohomology sequence of (2.4), i.e. the direct limit of the exact sequence:

$$
\longrightarrow H_{c}^{p}(U) \stackrel{j^{*}}{\longrightarrow} H^{p}(S) \stackrel{i^{*}}{\longrightarrow} H^{p}(S-U) \stackrel{d^{*}}{\longrightarrow} .
$$

Let $C^{*}(S, U)$ denote the subgrating of $C^{*}(S)$ ' of elements whose support dose not meet $U$. The derived cohomology ring is denoted as usual by $H^{*}(S, U)$. The quotient grating, $C_{S}^{*}(U)=C^{*}(S) / C^{*}(S, U)$ has derived cohomology ring isomorphic to $H^{*}(U)$.

Let $S$ be an open subset of a compact space $S^{\prime}$. Let $B=S^{\prime}-S$ and $U \in A(S)$. The sequence:

$$
\longrightarrow H^{p}\left(S^{\prime}-U, S-U\right) \stackrel{j^{*}}{\longrightarrow} H^{p}\left(S^{\prime}-U\right) \stackrel{i^{*}}{\longrightarrow} H^{p}(S-S) \stackrel{d^{*}}{\longrightarrow}
$$

is exact and the direct limit may be identified with

$$
\longrightarrow H^{p}\left(S^{\prime}, S\right) \stackrel{j^{*}}{\longrightarrow} H^{p}(B) \stackrel{i^{*}}{\longrightarrow} I^{p}(S) \stackrel{d^{*}}{\longrightarrow} H^{p+1}\left(S^{\prime}, S\right) \text {. }
$$

2.7. In the case of a polyhedral manifold $S^{\prime}$ with manifold boundary $B, H^{*}\left(S^{\prime}, S\right)=0$. Thus, $i^{*}: H^{*}(B) \rightarrow I^{*}(S)$ is a ring isomorphism. This could be used as a justification of the term "cohomology of the ideal boundary"'.

2.8. In developing the analogous concept of end homology groups it is necessary to follow the definition of Borel and Moore for the homology of a locally compact Hausdorff space $S$ [14]. As these seminar notes [14] are not yet readily available, we shall recall the definitions and some of the porperties of the homology groups. These homology groups are determined $(2.8,(2))$ by the Alexander-Spanier cohomology groups with compact support. They are used by Borel and Moore to obtain the Poincaré duality for generalized manifolds with an arbitrary principal ideal domain as coefficient domain, and in terms of a homology computable in terms of the cohomology. If $L$ is a field, the homology groups are isomorphic to the single space Čech homology groups of [6]; if $L$ is the integers then they may differ from the single space Čech homology groups.

Let $0 \rightarrow L \rightarrow A_{0} \rightarrow A_{1} \rightarrow 0$ be an injective resolution of $L,(L$ is hereditary). This permits one to regard $A=A_{0}+A_{1}$ as an augmented complex over $L$. Let $C=\operatorname{Hom}\left(C_{c}^{*}(S), A\right)$. Define $C_{p}(S)=$ Hom $\left(C_{c}^{p}(S), A\right) \oplus \quad \operatorname{Hom}\left(C_{c}^{p+1}(S), A_{1}\right) \quad$ with differential $\quad d f(c)=d(f(c))+$ $(-1)^{p+1} f(d c), f \in C_{p}, c \in C_{c}^{*}(S)$. It is shown in [14] that:

(1) $C_{*}(S)$ is a complete grating

(2) for each open $U \subset S$, the sequence 
$0 \longrightarrow \operatorname{Ext}\left(H^{p+1}\left(C_{c}^{*}(U)\right), L\right) \longrightarrow H_{p}\left(\operatorname{Hom}\left(C_{c}^{*}(U), A\right)\right.$

$\longrightarrow \operatorname{Hom}\left(H^{p}\left(C_{c}^{*}(U)\right), L\right) \longrightarrow 0$, is exact and splits.

(3) The above exact sequence is compatible with homomorphisms.

(4) The associated sheaf is flabby (flasque) (or $\phi-$ fine, if $\phi$ is a paracompactifying family).

2.9. The homology group $H_{p}(S ; L$ ( $L$ usually suppressed) is defined to be $H_{p}\left(\operatorname{Hom}\left(C_{c}^{*}(S), A\right)\right)$.

2.10. If $U$ is an open subset of $S$ then $C_{c}^{*}(U)$, and $C_{c}^{*}(S) / C_{c}^{*}(U)$ can be used to define the homology groups $H_{*}(U ; L)$ and $H_{*}(S-U ; L)$ respectively, [14]. We obtain the exact sequence:

$$
\longrightarrow H_{p}(S-U) \stackrel{i_{*}}{\longrightarrow} H_{p}(S) \stackrel{j_{*}}{\longrightarrow} H_{p}(U) \stackrel{d_{*}}{\longrightarrow} H^{p-1}(S-U) \longrightarrow
$$

as the derived homology sequence of exact ( $A$ is injective) sequence:

$$
\begin{aligned}
0 \longrightarrow \operatorname{Hom}\left(C_{c}^{*}(S) / C_{c}^{*}(U), A\right) \longrightarrow \operatorname{Hom}\left(C_{c}^{*}(S), A\right) \\
\longrightarrow \operatorname{Hom}\left(C_{c}^{*}(U), A\right) \longrightarrow 0 .
\end{aligned}
$$

2.13. The group $H_{p}^{c}(S)$ will denote the pth homology of those chains $C_{p}^{c}(S)$ of $C_{p}(S)$ with compact support. (The support of a chain is defined analogously to that in [1], or equivalently, as the support of the cross section which represents the given chain in the associated sheaf of chains.) The subgrating $C_{p}^{c}(S)$ may be identified with the direct limit of $C_{p}(\bar{V})=\operatorname{Hom}\left(C_{c}^{*}(S) / C_{c}^{*}(S-V), A\right)$ taken over all $V \in A(S)$. This follows from the fact that the map $\operatorname{Hom}\left(C_{c}^{*}(S) / C_{c}^{*}(S-M), A\right) \rightarrow$ Hom $\left(C_{c}^{*}(S) / C_{c}^{*}(S-N), A\right)$ is an injection for all compact $M, N, M \subset N$ $\subset S$. Thus, $H_{p}^{c}(S)$ may be identified with the direct limit of $H_{p}(M)$, for all compact $M \subset S$.

We shall define $H_{p}^{c}(S, U)$ to be $H_{p}\left(C_{*}(S) / C_{*}^{c}(U)\right)$. This is identified, therefore, with the direct limit, over all compact $M \subset U$, of $H_{p}(S-M)$.

As the sequence:

$$
0 \longrightarrow C_{p}^{c}(S) \stackrel{i_{*}}{\longrightarrow} C_{*}(S) \stackrel{i_{*}}{\longrightarrow} C_{*}(S) / C_{*}^{c}(S) \longrightarrow 0
$$

is exact and the boundary operator decreases the supports we can obtain the derived exact sequence:

$$
\longrightarrow H_{p}^{c}(S) \stackrel{i_{*}}{\longrightarrow} H_{p}(S) \stackrel{j_{*}}{\longrightarrow} I_{p}(S) \stackrel{d_{*}}{\longrightarrow} H_{p-1}^{c}(S) \longrightarrow
$$

Clearly, $I_{p}(S)=H_{p}\left(C_{*}(S) / C_{*}^{c}(S)\right)$ identifies itself with the direct limit over the compact sets $M \subset S$ of $H_{p}(S-M)$. In particular, $I_{p}(S)=$ direct limit over $V \in A(S)$ of $H_{p}(S-\bar{V})$. For a locally finite polyhedron $S, I_{*}(S)$ is isomorphic to the homology of the infinite chains mod the finite chains. (The term "homology of the ideal boundary" for $I_{*}(S)$ may not be justified as in the case for cohomology 
because if $S^{\prime}$ were a polyhedral manifold with boundary $B, S=S^{\prime}-B$, then $I_{p+1}(S)=H_{p}(B)$, (cf. 2.7). That is, one might expect from the name we have chosen, that $I_{p}(S)=H_{p}(B)$. But the decision to call $I_{*}(S)$ the homology of the ideal boundary of $S$ rests on the similarity, on the chain level, of the definitions of cohomology of the ideal boundary and the results of $\S 3$.)

2.16. THEOREM. Let $S \subset S^{\prime}$ as an open subset of the locally compact Hausdorff space $S^{\prime}$. Then the sequence:

$$
\longrightarrow H_{p}\left(S^{\prime}-S\right) \stackrel{i_{*}}{\longrightarrow} H_{p}^{c}\left(S^{\prime}, S\right) \stackrel{j_{*}}{\longrightarrow} I_{p}(S) \stackrel{d_{*}}{\longrightarrow} H_{p-1}\left(S^{\prime}-S\right) \longrightarrow
$$

is exact.

Proof. Let $M$ be a compact set $\subset S$. Then consider the exact sequence of chains:

$$
0 \longrightarrow C_{p}\left(S^{\prime}-S\right) \longrightarrow C_{p}\left(S^{\prime}-M\right) \longrightarrow C_{p}(S-M) \longrightarrow 0
$$

Passing to the direct limit over all compact $M \subset S$ of the derived homology sequence of (2.17) we obtain by use of the identification discussed above the desired result.

2.18. Observe that if $S^{\prime}$ denotes the one point compactification of $S, j_{*}: H_{p}^{c}\left(S^{\prime}, S\right) \rightarrow I_{p}(S)$ is an isomorphism, $p>0$. The group $H_{p}^{c}\left(S^{\prime}, S^{\cdot}\right.$ in this case is precisely the local homology group $S^{\prime}$ at $\infty\left(\infty=S^{\prime}-S\right)$ )

3. Poincaré duality for the ideal boundary. We shall adhere to the terminology of [9] and [1] for generalized $n$-manifolds ( $n$-gm's). The ring of coefficients is still the principal ideal domain, $L$.

3.1. Theorem. Let $X$ be a paracompact orientable $n$-gm. Then, the diagram

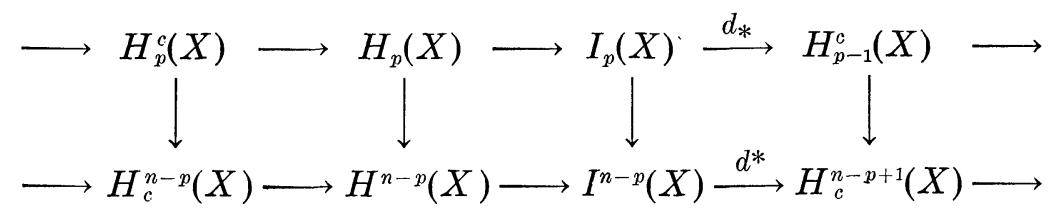

is commutative. The horizontal rows are exact and the vertical maps are isomorphisms (Poincaré duality).

Proof. This may be obtained from the five lemma by just defining a natural map from $C_{p}(X) / C_{p}^{c}(X)$ to $C^{n-p}(X) / C_{c}^{n-p}(X)$ by means of a map induced from Poincaré duality for orientable n-gm's. with compact and closed support, respectively.

An alternate procedure would be to let $U \in A(X)$ and to consider the commutative diagram 


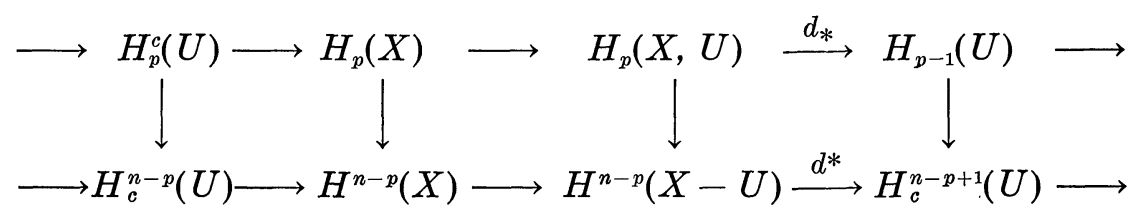

The group $H_{p}(X, U)$ is computed by taking the homology of the chains of $X \mathrm{mod}$ those chains of $X$ whose support (closed in $X$, hence, compact in $U$ ) lie in $U$. Each vertical map is an isomorphism. (This explicit form of the Poincaré duality theorem for homology manifolds, discussed in detail in the author's dissertation, University of Michigan, 1958, is derived from [2; Ch. 19, Th. 7].) Now by passing to the direct limit over all $U \in A(X)$ we obtain the desired reslut.

A Poincaré duality theorem for non-orientable manifolds may be obtained if twisted coefficients are used.

\section{The end point compactification of generalized manifolds.}

4.1. Let $S$ be an open subset of a compact Hausdorff space $S^{\prime}$. Let $B=S^{\prime}-S$ be the union of a finite number of closed disjoint sets $B_{i}$. Let $V_{i}$ be regular open sets (1.8) about $B_{i}$ such that $\bar{V}_{i} \cap \bar{V}_{j}=\phi$, $i \neq j$. Assume, furthermore, that no component of $V_{\imath}-B_{i}$ is a bounded set of $S$. Let $V=\bigcup_{i=1}^{h} V_{i}$, then $S^{\prime}-V=\bar{U}, U \in A(S)\left(V_{i}\right.$ are regular open sets). Consider the exact sequence:

$$
\longrightarrow H_{p}(B) \longrightarrow H_{p}(S-\bar{U}) \longrightarrow H_{p}(S-\bar{U}) \stackrel{d_{*}}{\longrightarrow} H_{p-1}(B) .
$$

The sequence (a) splits into to direct product of the exact sequences:

$$
\longrightarrow H_{p}\left(B_{i}\right) \longrightarrow H_{p}\left(V_{i}\right) \longrightarrow H_{p}\left(V_{i}-B_{i}\right) \stackrel{d_{*}}{\longrightarrow} H_{p-1}\left(B_{i}\right),
$$

because $C_{c}^{*}(V)=\sum_{i} C_{c}^{*}\left(V_{i}\right), C_{c}^{*}(V-B)=\sum_{i} C_{c}^{*}\left(V_{i}-B_{i}\right), C_{c}^{*}(V) / C_{c}^{*}(V-B)$ $=\sum_{i}\left(C_{c}^{*}\left(V_{i}\right) / C_{c}^{*}\left(V_{i}-B_{i}\right)\right)$. The set of $U$ of the above type is cofinal in $A(S)$. Thus,

4.2. Lemma. In passing to the direct limit over the $U$ of the above type, sequence (a) becomes the exact sequence:

$$
\longrightarrow H_{p}(B) \longrightarrow H_{p}^{c}\left(S^{\prime}, S\right) \longrightarrow I_{p}(S) \stackrel{d_{*}}{\longrightarrow} H_{p-1}(B),
$$

which splits into the direct product of the exact sequences:

$$
\longrightarrow H_{p}\left(B_{i}\right) \longrightarrow H_{p}^{c}\left(S^{\prime}, S^{\prime}-B_{i}\right) \longrightarrow I_{p}\left(S^{\prime}-B_{i}\right) \stackrel{d_{*}}{\longrightarrow} H_{p-1}\left(B_{i}\right) .
$$

4.3. REMARK. The above lemma points out that the group $I_{p}(S)$ is determined "locally at infinity".

4.4. Lemma. Let $S^{\prime}$ be a connected, compact Hausdorff space, $B$ a closed totally disconnected subset of $S^{\prime}$. Let $S=S^{\prime}-B$ have finite 
cohomology dimension (in the sense of Cohen [3]), and be clc [1 or 12]. Then, $S^{\prime}$ has finite cohomology dimension and is clc, if and only if, $H^{*}\left(S^{\prime}\right)$ is finitely generated.

Proof. The dimension of $B$ is 0 , as $B$ is totally disconnected. The sum theorem then implies that $S^{\prime}$ must have finite cohomolgy dimension. Because $B$ is totally disconnected, $S^{\prime}$ compact, connected, and $S$ is locally connected, $S^{\prime}$ must be locally connected (see 1.8).

Suppose that $H^{*}\left(S^{\prime}\right)$ is finitely generated. Let $x \in B$ and $P_{x}$ be any open neighborhood of $x$. In the argument of Theorem 1.8 it was seen that a closed neighborhood of $x$ say $C_{1}, C_{1} \subset P_{x}$, and a closed neighborhood $C_{2}$ of $B-\left(C_{1} \cap B\right)$ could be chosen so that $C_{1} \cap C_{2}=\phi$. Let $C=C_{1} \cup C_{2}$. Let $D_{1}$ be a smaller closed neighborhood of $C_{1} \cap B$ and $D_{2}$ of $C_{2} \cap B$. Set $D=D_{1} \cup D_{2}$. The closure of $S^{\prime}-C$ can be chosen to be contained in $S^{\prime}-D$, contained within $S$. The clc of $S$ implies that the image of $H_{c}^{*}\left(S^{\prime}-C\right)$ in $H_{c}^{*}\left(S^{\prime}-D\right)$ is finitely generated. Since $H^{*}\left(S^{\prime}\right)$ is also finitely generated it follows that the image of $H^{*}(C)$ in $H^{*}(D)$ must be finitely generated. In particular, the image of $H^{*}\left(C_{1}\right)$ in $H^{*}\left(D_{1}\right)$ is finitely generated. The direct limit of the cohomology groups of a closed neighborhood system of $x$ must equal the cohomology of the point $x$. Thus, one can choose a sufficiently small neighborhood $Q_{x}$ such that $H^{p}\left(\bar{P}_{x}\right) \rightarrow H^{p}\left(\bar{Q}_{x}\right)$ is trivial, for $p>0$. Therefore, $S^{\prime}$ is clc.

If $S^{\prime}$ is clc and has finite cohomology dimension then it is known that $H^{*}\left(S^{\prime}\right)$ is finitely generated, [5].

4.5. THEOREM. Let $X$ be an orientable generalized $n$-manifold. Then, $X^{\prime}$, the end point compactification of $X$ is an orientable generalized $n$-manifold, if and only if, $I_{p}(X)=0 ; p \neq 1, n, n>1$, and $H^{*}\left(X^{\prime}\right)$ is finitely generated.

Proof. The exact sequence of the Theorem 2.16 becomes in the case at hand:

$$
\longrightarrow H_{p}\left(X^{\prime}-X\right) \stackrel{i_{*}}{\longrightarrow} H_{p}^{c}\left(X^{\prime}, X\right) \stackrel{j_{*}}{\longrightarrow} I_{p}(X) \stackrel{d_{*}}{\longrightarrow} H_{p-1}\left(X^{\prime}-X\right) .
$$

If $X^{\prime}$ is an orientable $n$-gm then Poincaré duality implies that $H_{p}^{c}\left(X^{\prime}, X\right)$ $=H^{n-p}\left(X^{\prime}-X\right)=0, p \neq n$, (See the proof of 3.1 for the form of Poincaré duality used.) Thus, $I_{p}(X)=0, p \neq n, 1$.

Suppose $I_{p}(X)=0, p \neq n, 1$ and $H^{*}\left(X^{\prime}\right)$ is finitely generated. To show that $X^{\prime}$ is an orientable $n$-gm consists of first showing that the local homology groups are "correct" in a manner that is sufficient to show that $X^{\prime}$ is an orientable homology n-manifold. Then, Poincaré duality enables one to transform the clc condition into the "correct" local cobetti numbers.

Let $x \in X^{\prime}-X=B$ and $U_{x}$ be any neighborhood of $x$. In the proof of 1.8 it was seen that an open neighborhood of $x, O_{x}$, and an open neighborhood $O_{x}^{\prime}$ of $B-\left(O_{x} \cap B\right)$ could be chosen so that $O_{x}^{\prime} \cap O_{x}$ 
$=\phi$, and $\bar{O}_{x} \subset U_{x}$. Let $O=O_{x} \cup O_{x}^{\prime}$. Furthermore, $O$ could have been chosen so that it was a regular open set with $O_{x}$ consisting of a single component and $O_{x}^{\prime}$ such that each of its components meet $B$. Note that $O$ has at most a finite number of components. Choose $Q$ with properties similar to $O$ and such that $\bar{Q}_{x} \subset O_{x}, \bar{Q} \subset O$. The image $H^{*}\left(X^{\prime}-Q\right) \stackrel{i^{*}}{\longrightarrow}$ $H^{*}\left(X^{\prime}-O\right)$ is finitely generated as the image $H_{c}^{*}(Q) \stackrel{j^{*}}{\longrightarrow} H_{c}^{*}(O)$ is finitely generated, (4.4) and $H^{*}\left(X^{*}\right)$ is finitely generated. Now the universal coefficient Theorem $(2.8,(2))$ is compatible with homomorphisms induced by the inclusion $X^{\prime}-O \subset X^{\prime}-Q$. For, $\operatorname{Hom}\left(C^{*}\left(X^{\prime}\right) / C_{c}^{*}(Q), A\right)$ and Hom $\left(C^{*}(X) / C_{c}^{*}(O), A\right)$ can be regarded as chains of $X^{\prime}-O$ and $X^{\prime}-Q$, respectively. In $\S 2.13$ it was seen that the inclusion induces an injection of the chains of $X^{\prime}-O$ into those of $X^{\prime}-Q$. Thus conditions are satisfied for the commutativity of:

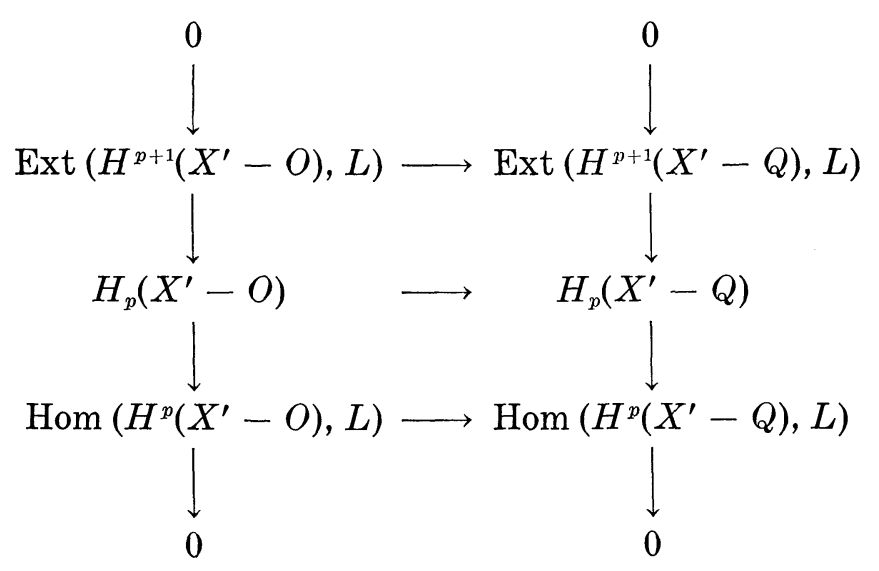

By choosing an open set $W$ with similar properties as $O$ and $Q$ such that, $\bar{O} \subset W \subset \bar{W} \subset O, \bar{Q}_{x} \subset W_{x} \subset \bar{W}_{x} \subset O_{x}$, and inserting the universal coefficient sequence of $X^{\prime}-W$ between the two columns of the above diagram it follows that the image of $H_{p}\left(X^{\prime}-O\right)$ in $H_{p}\left(X^{\prime}-Q\right)$ is finitely generated. Since $H^{*}\left(X^{\prime}\right)$ is finitely generated, the image of $H_{*}(O)$ in $H_{*}(Q)$ must be finitely generated. Since $H_{*}(O)$ and $H_{*}(Q)$ splits into the direct product of homologies on each compoment (4.1), the image of $H_{*}\left(O_{x}\right)$ in $H_{*}(Q)$ must be finitely generated. (We are not saying that the mapping induced by the inclusion splits into a direct product.) We can choose a neighborhood system of sets like the $O$ and the $Q$ of above which are cofinal in $A(X)$ when $B$ is deleted and with the component containing $x$ being a cofinal neighborhood system of $x$. Therefore, as $I_{p}(X)=0$, $p \neq 1, n$ we may find a $Q_{x}$, for $U_{x}$ such that the image of $H_{p}\left(U_{x}\right)$ in $H_{p}\left(Q_{x}\right)$ is trivial, for $p \neq 1, n$. Hence, the local homology groups of $x$ vanish in dimensions different from $n$ and 1.

We need the fact that $X^{\prime}$ is $l c^{\infty}$ in the sense of homology. Let $x \in X^{\prime}$, and $U$ be any open neighborhood of $x$. One can find $V$ and 
$W$ such that $H^{p}(\bar{U}) \longrightarrow H^{p}(\bar{V})$ and $H^{p}(\bar{V}) \longrightarrow H^{p}(\bar{W})$ are trivial, since $X^{\prime}$ is clc. A diagran similar to (4.6) involving $H_{p}(\bar{W}), H_{p}(\bar{V})$ and $H_{p}(\bar{U})$ implies that $H_{p}(\bar{W}) \longrightarrow H_{p}(\bar{U})$ is trivial. Hence $X^{\prime}$ is $l c^{\infty}$.

In particular, the space $X^{\prime}$ is $l$-lc. Let $x \in X^{\prime}$ and $V$ and $U$ be open connected sets about $x$. Then as $n>1$, or as $x$ may be an element of $X^{\prime}-X, \bar{V}-x$ and $\bar{U}-x$ are still connected. Thus, $H_{0}^{c}(\bar{V}-x)$ $\longrightarrow H_{0}(\bar{V})$ and $H_{0}^{c}(\bar{U}-x) \longrightarrow H_{0}(\bar{U})$ are isomorphisms. Choose $V$ sufficiently small so that $H_{1}(V) \longrightarrow H_{1}(\bar{U})$ is trivial. Consider the commutavive diagram:

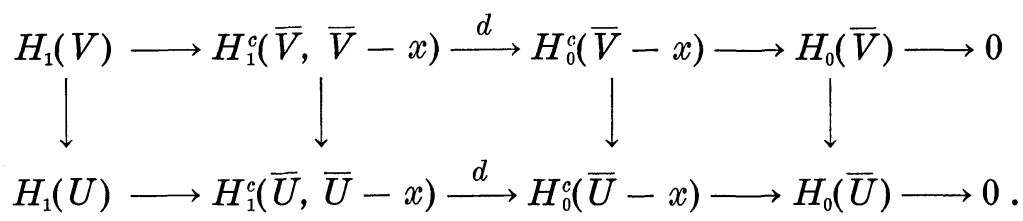

The horizontal rows are exact, of course, and all maps except the boundary maps are induced by inclusions. By the above remarks, the first vertical map and the boundary maps are trivial. Therefore, the second vertical map is also trivial. But the second vertical map is an isomorphism onto, as can be readily seen from the definition of the relative groups $(\S 2)$. By the same token $H_{1}^{c}(\bar{V}, \bar{V}-x) \approx H_{1}^{c}\left(X^{\prime}, X^{\prime}-x\right)=0$, i.e. the local homology group in dimension 1 vanishes.

In dimension $n, H_{c}^{n}(X) \rightarrow H^{n}\left(X^{\prime}\right)$ is an isomorphism. In fact, for any connected open set $U$ of $X^{\prime}, U-\left(X^{\prime}-X\right)$ is still connected and $H_{c}^{n}\left(U-\left(X^{\prime}-X\right)\right) \rightarrow H_{c}^{n}(U)$ is an isomorphism. This clearly implies that $H_{c}^{n}(U) \rightarrow H_{c}^{n}\left(X^{\prime}\right)$ is an isomorphism, and is isomorphic to $L$. Thus, $X^{\prime}$ is an orientable homology $n$-manifold. Consequently, Poincaré duality, holds.

In order to show that $X^{\prime}$ is an orientable $n$-gm we still need to show that $p^{r}\left(x ; X^{\prime}\right)=0, r \neq n$, all $x \in X^{\prime}-X$. Let $x \in X^{\prime}$ and $U$ any open set containing $x$. We can find an open set $V$ such that $H_{p}^{c}(V) \rightarrow H_{p}^{c}(U)$ is trivial, $p \neq 0$, since $X^{\prime}$ is $l c_{\infty}$. By Poincaré duality (compact supports) $H_{c}^{n-p}(V) \rightarrow H_{c}^{n-p}(U)$ must be trivial, $p \neq 0$. This completes the proof of the theorem.

4.7. COROLlaRIES. Let $X$ be an orientable generalized $n$-manifold such that $H_{c}^{*}(X) \cong H_{c}^{*}\left(\left(E^{n}\right)\right.$, where $F^{n}$ is Euclidean $n$-space. Then, the one point compactification of $X$ is an orientable generalized $n$ manifold with cohomology isomorphic to the cohomology of the n-sphere.

This assertion is an immediate consequence of 4.5. However it also may be deduced directly. The calculation of the local homology groups at infinity is considerably simplified because one need not make any recourse to the homology of the ideal boundary when $H_{c}^{r}(X)=0$, $r \neq n$, and $H_{c}^{r}(X)=H^{r}\left(S^{n-1}\right)$. Furthermore, in this case, it is easy 
to see directly that $X^{\prime}$, is clc at infinity. As in 4.5 , one can then proceed from an orientable homology $n$-manifold to an orientable $n$-gm. R. L. Wilder has informed me that he also has obtained a proof of this corollary when $L$ is a field.)

The one point compactification of any orientable generalized $n$ manifold $X$ is an orientable generalized $n$-manifold, if and only if, $X$ has one end, $H_{c}^{*}(X)$ is finitely generated and $I_{p}(X)=0, p \neq 1, n$, $n<1$.

One the other hand, the end point compactification of an orientable paracompact $n$-gm $X$ is an orientable $n$-gm with cohomology isomorphic to the $n$-sphere, if and only if, $H_{c}^{p}(H)=0, p \neq 1, n$, and $H^{*}\left(X^{\prime}\right)$ is finitely generated.

4.8. Remark. By 4.4, the condition that $H^{*}\left(X^{\prime}\right)$ is finitely generated may be replaced by the equivalent condition that $X^{\prime}$ is clc.

4.9. REMARK. If $H_{c}^{*}(X)$ is finitely generated, then $H^{*}\left(X^{\prime}\right)$ is finitely generated and there exists at most a finite number of ends.

4.10. If $X$ were paracompact then the condition $I_{p}(X)=0, p \neq 1$, $n,(n>1)$ may be replaced by the equivalent condition $I^{p}(X)=0$, $p \neq 0, n-1,(n>1)$, by 3.1 .

4.11. If $X$ were paracompact and $L$ were a field then $I_{p}(X)=0$, $p \neq 1, n$ implies that $H_{c}^{p}(X)$ is finitely generated $p \neq 1$. For if $H_{c}^{p}(X)$ were not finitely generated then $H_{n-p}(X)$ would be uncountably generated. Since $H_{c}^{*}(X)$ is at most countably generated this would imply that $I^{p}(X)$ could not be trivial. Thus, in this case, the condition $H^{*}\left(X^{\prime}\right)$ be finitely generated may be replaced by the condition that $H^{1}\left(X^{\prime}\right)$ be finitely generated.

4.12. The ends of the end point compactification of $P^{2} \times E^{1}$, where $P^{2}$ denotes the real projective plane and $E^{1}$ the real line consists of exactly 2 points. If $L$ equals the rationals then $I_{2}(X)=0$. However, the compactification is not a 3 -gm over $L$. This example shows the necessity of the orientability hypothesis in 4.5. To obtain a theorem valid for locally orientable $n$-gm's we need to make an assumption about the groups $I_{n}(X)$.

4.13. THEOREM. Let $X$ be a locally orientable $n$-gm such that $H^{*}\left(X^{\prime}\right)$ is finitely generated. Then the end point compactification $X^{\prime}$ of $X$ is a locally orientable $n$-gm, if and only if,

$$
\begin{aligned}
& I_{p}(X)=0, \text { for } p \neq 1, n \\
& I_{n}\left(X^{\prime}-x\right)=L, \text { for each } x \in X^{\prime}-X .
\end{aligned}
$$

Proof. The arguments of 4.5 are local except in dimension $n$. Furthermore, the use of the Poincaré duality in the proof of 4.5 is again only local. 
In dimension $n$ we need to select a connected open neighborhood $O_{x}$ of $x \in X^{\prime}-X$ such that we can find a connected open neighborhood $Q_{x}$ of $x$ so that the image of $H_{n}\left(O_{x}\right)$ in $H_{n}\left(Q_{x}\right)$ is isomorphic to $L$. This is possible by the methods of 4.5 together with the assumption of the theorem. By exactness, the image of $H_{n}\left(O_{x}-B\right)$ in $H_{n}\left(Q_{x}-B\right)$ is isomorphic to the image of $H_{n}\left(O_{x}\right)$ in $H_{n}\left(Q_{x}\right)$. This is possible, if and only if, $H_{c}^{n}\left(O_{x}-B\right) \cong L$, as $O_{x}-B$ is connected, and $X$ locally orientable. Thus, $X^{\prime}$ must be a locally orientable $n$-gm.

\section{Compactification of open (locally Euclidean) 2 manifolds.}

5.1. All open and closed separable 2-manifolds can be constructed by pasting together 5 elementary figures; see for example [8: Chap. 5] (while the methods described are not precisely those of the Froudenthal compactification they are very closely related). Theorem 4.5 makes it quite easy to see to which type connected open 2-manifolds belong:

(a) those open 2-manifolds which are homeomorphic to a closed 2manifold minus a closed totally disconnected set;

(b) those open 2-manifolds which cannot be so represented.

Whether a given 2-manifold is of class (a) or (b) simply depends on whether $H^{1}\left(X^{\prime}\right)$ is finitely generated or not (use integers mod 2 if $X$ is not orientable and rationals if it is). The group $H_{1}\left(X^{\prime} ; Z\right)$ completely determines the closed 2-manifold $X^{\prime}$. If $H^{1}\left(X^{\prime}\right)$ is finitely generated then $X$ is a sphere with a certain number of handles and cross-caps (determined by $H_{1}\left(X^{\prime}\right)$ ), with a totally disconnected set of points deleted. Obviously, if $H_{c}^{1}(X)$ had been finitely generated, then $X$ is homeomorphic to a 2-manifold with boundary deleted. This is obtained by replacing each end point of $X$ by a closed disk. As $X^{\prime}$ is a closed 2manifold, this can be done.

5.2. To find a compact 3 -manifold $X^{*}$ with boundary $B^{*}$ for a given locally Euclidean 3-manifold $X$ with finitely generated $H_{c}^{*}(X)$ is, in general, impossible. For example, if $X$ were the bad complement of the Alexander-horned sphere (in the 3 -sphere) then its boundary $B^{*}$, if it exists, must be a 2-sphere (2.7). But arbitrarily close to this boundary there would be small circles which could not be shrunk to a point in the complement of the boundary. However, close to the boundary the compactification (as a manifold with boundary) would be homeomorphic to $S^{2} \times I^{1}, S$ the 2 -sphere and $I^{1}$ the unit interval. Thus such a compactification could not exist.

If one were to take the example of Whitehead [11] of an open contractible subset of Euclidean 3-space then a compactification to a 3-manifold with boundary is also impossible.

Of course, in these two examples the one point compactification is a spherelike $3-\mathrm{gm}$. This is a special case of the fact that the end 
point compactification $X^{\prime}$ of any orientable paracompact 3 -gm $X$ with $H_{c}^{2}(X ; L)=0$ is a spherelike orientable $3-\mathrm{gm}$. This follows from the third paragraph of 4.7. (To see that $H^{*}\left(X^{\prime}\right)$ is finitely generated one could use 1.13.)

\section{Mappings of manifolds.}

6.1. Theorem. Let $f: X \rightarrow X^{*}$ be the mapping which collapses each component of the boundary $B$ of the compact connected orientable $n$-gm $X$ with boundary to a distinct point. Then $X^{*}$ is an orientable $n$-gm, if and only if, each component of $B$ is an orientable spherelike $(n-1)-\mathrm{gm}$.

Proof. We adhere to the terminology of [9] for $n$-gm's with boundary. Let $D X$ denote the double of $X$, then $I_{p}(D X-B) \cong I_{p}(X-B)$ $\oplus I_{p}(X-B)$. By [9; 2.3], $D X$ is an orientable $n$-gm and $X-B$ is connected by $[9 ; 3.3]$. By $[9 ; 3.3]$ each component $B_{i}$ correspends to an end point of $X$, hence $X^{*}=X^{\prime}$. Therefore, using (4.5), $X^{*}$ is an orientable $n$-gm, if and only if, $I_{p}(X-B)=0$. Let $n>2$, and consider the exact sequence:

$0 \longrightarrow H^{0}(B) \longrightarrow I_{n}(D X-B) \longrightarrow H_{n-1}(B) \longrightarrow H^{1}(B) \longrightarrow I_{n-1}(D X-B)$

$\longrightarrow H_{n-2}(B) \longrightarrow H^{2}(B) \longrightarrow I_{n-2}(D X-B) \longrightarrow \cdots \longrightarrow H_{1}(B) \longrightarrow H^{n-1}(B)$.

The sequence is nothing but (2.16) where we have identified $H_{p}^{c}(D X$, $D X-B)$ with $H^{n-p}(B)$, by Poincaré duality. Since $H^{j}(B)=H_{(n-1)-j}(B)$, and $H^{1}(B)$ is torsion free when $L$ is not a field, it follows that $H_{j}(B)=0$, $j \neq 0, n-1$, if and only if, $X^{*}$ is an orientable $n$-gm. This completes the proof.

6.2. We remark that the above theorem would be an immediate corollary of the vanishing of $H_{*}^{c}(X, X-B)$ if this were known for $n$ gm's with boundary. In fact this would imply a Poincare duality of the duality of the Lefschetz type $H_{p}(X) \cong H_{c}^{n-p}(X-B)$ involving the homology of the total manifold with boundary $X$, and in additions $i^{*}: H^{*}(B) \rightarrow I^{*}(X-B)$ would be a ring isomorphism as in 2.7.

6.3. In [13], Wilder has shown that an $(n-1)$-monotone image of a compact orientable $n$-gm onto a Hausdorff space is again a compact orientable $n-\mathrm{gm}$ with isomorphic homology groups. The coefficient domain was a field. Using the spectral sequence of a map one can prove this theorem for a general principal ideal domain, $L$. Little information is given however in the case the map is only $(n-2)$-monotone. We may use the end theory in the special case that all but a finite number of inverse images of points in the image space is acyclic.

Let $X$ be a compact connected orientable $n$-gm. Let $B$ be a closed 
subset such that $H^{*}(B) \cong H^{*}\left(S^{n-1}\right)$. Then the end point compactification of $X-B$ consists of two points. If $B$ separates $X$ then $(X-B)^{\prime}$ is the union of two disjoint orientable $n$-gm's each compactified by one end. If $B$ fails to separate $X$ then $(X-B)^{\prime}$ is an orientable $n$-gm compactified by 2 ends. The one point campactification of $X-B$ corresponds to identifying the two ends to a single point, or equivalently collapsing $B$ to a single point.

Let $B-\bigcup_{i=1}^{k} B_{i}$ be the union of disjoint closed subsets of a compact connected orientable $n$-gm $X$ such that $H^{*}\left(B_{i}\right) \cong H^{*}\left(S^{n-1}\right)$. Let $f: X \rightarrow Y$ be the map which collapses each point $B_{i}$ to a point $y_{i}$. Factor $f$, by $g_{1}: X \rightarrow Y_{1}$, by first collapsing only the $B_{i}$ (say $B_{1}, B_{2}, \cdots$, $\left.B_{s}\right)$, which separate $X$. Then $Y_{1}$ is the connected union of $(S+1)$ connected orientable $n$-gm's each two of which are either disjoint or meet in a point $y_{1}, y_{2}, \cdots$, or $y_{s}$. Each $y_{i}, i \leqq s$, is a cut point of $Y_{1}$. The map $g_{2}$ defined by $g_{2} \circ g_{1}=f$ collapses the remaining $B_{s+1}, \cdots, B_{k}$ to the points $y_{s+1}, \cdots, y_{k}$. Each $B_{s+j}$ lies in one of the orientable $n$-gm's of $Y_{1}$ and does not meet any of the cut points. The $B_{s+\rho}$ fail to separate the $n$-gm's and so collapsing the $B_{s+j}$ to a point $y_{s+j}$ corresponds to first removing the $B_{s+j}$, then compactifying by the end point compactification to obtain an orientable $n$-gm, and then collapsing the two end points to a single point.

Now suppose in the original compact orientable connected $n$-gm $X$ there is a map $g$ from $X$ to a Hausdorff space $X^{*}$ such that the inverse image of each point of $X^{*}$ is acyclic except for a finite number of points $x_{1}^{*}, \cdots, x_{k}^{*}$. Suppose $H^{*}\left(g^{-1}\left(x_{i}^{*}\right)\right) \cong H^{*}\left(S^{n-1}\right)$. Then $g$ may be factored by the map $f$ of the above paragraph. Define $h: Y \rightarrow X^{*}$ by $h \circ g_{2} \circ g_{1}=g$. As the inverse image of each point of the map $h$ is acyclic the space $X^{*}$ has the same cohomology structure as $Y$. Furthermore, each orientable $n$-gm of $Y$ or orientable $n$-gm of $Y$ with identifications goes by $h$ into a corresponding $n$-gm of $X^{*}$ with the same cohomology structure.

\section{REFERENCES}

1. A. Borel, The Poincaré duality in generalized manifolds, Mich. Math. Jour., 4, no. 3 (1957), 227-241.

2. H. Cartan, Séminaire Henri Cartan, 3e année 1950/1951 Paris (1955).

3. H. Cohen, A cohomological definition of dimension for locally compact Hausdorff spaces, Duke Math. Jour., 21 (1954), 209-224.

4. P. Conner, On the impossibility of fibering certain manifolds by a compact fiber, Mich. Math. Jour., 4, no. 3 (1957), 241-249.

5. E. Dyer, Regular mappings and dimension, Annals of Math., 67, no. 1 (1958), 119-149.

6. S. Eilenberg and N. Steenrod, Foundations of algebraic topology, Princeton, 1952.

7. H. Freudenthal, Über die Enden topologischer Räume und Gruppen, Math, Zeitschrift, 33 (1931), 692-713. 
8. B. Kerékjártó, Vorlesungen über Topologie, Berlin, Springer, 1923.

9. F. Raymond, Separation and union theorems for generalized manifolds with boundary, Mich. Math. Jour. 7 (1960) pp. 7-21.

10. E. Specker, Die Erste Cohomologie gruppe von Überlagerungen und Homotopieegenschaften drei dimensionaler Mannigfaltigkeiten, Comm. Math. Helv., 23 (1949), 303-333. 11. J. H. C. Whitehead, A certain open manifold whose group is unity, Quart. Jour. of Math. (Oxford) 6 (1935), 268-279.

12. R. L. Wilder, Topology of Manifolds, Amer. Math. Soc. Colloquium Publications 32 (1949).

13. - Monotone mappings of manifolds, Pacific J. Math. 7 (1957), 1519-1528.

14. Lectures of a seminar in transformation groups, The Institute for Advanced Study, Princeton, N. J.

The Institute For AdVANCED Study 



\section{PACIFIC JOURNAL OF MATHEMATICS}

\section{EDITORS}

\author{
David Gilbarg \\ Stanford University \\ Stanford, California \\ F. H. Brownell \\ University of Washington \\ Seattle 5 , Washington
}

\author{
A. L. Whiteman \\ University of Southern California \\ Los Angeles 7, California \\ L. J. PAIGE \\ University of California \\ Los Angeles 24, California
}

\section{ASSOCIATE EDITORS}

\author{
E. F. BECKENBACH \\ T. M. CHERRY \\ D. DERRY
}
E. HEWITT
A. HORN
L. NACHBIN
M. OHTSUKA
H. L. ROYDEN
M. M. SCHIFFER

E. SPANIER

E. G. STRAUS

F. WOLF

\section{SUPPORTING INSTITUTIONS}

\author{
UNIVERSITY OF BRITISH COLUMBIA \\ CALIFORNIA INSTITUTE OF TECHNOLOGY \\ UNIVERSITY OF CALIFORNIA \\ MONTANA STATE UNIVERSITY \\ UNIVERSITY OF NEVADA \\ NEW MEXICO STATE UNIVERSITY \\ OREGON STATE COLLEGE \\ UNIVERSITY OF OREGON \\ OSAKA UNIVERSITY \\ UNIVERSITY OF SOUTHERN CALIFORNIA
}

\author{
STANFORD UNIVERSITY \\ UNIVERSITY OF TOKYO \\ UNIVERSITY OF UTAH \\ WASHINGTON STATE COLLEGE \\ UNIVERSITY OF WASHINGTON

AMERICAN MATHEMATICAL SOCIETY
CALIFORNIA RESEARCH CORPORATION
HUGHES AIRCRAFT COMPANY
SPACE TECHNOLOGY LABORATORIES
NAVAL ORDNANCE TEST STATION

Mathematical papers intended for publication in the Pacific Journal of Mathematics should be typewritten (double spaced), and the author should keep a complete copy. Manuscripts may be sent to any one of the four editors. All other communications to the editors should be addressed to the managing editor, L. J. Paige at the University of California, Los Angeles 24, California. ..

50 reprints per author of each article are furnished free of charge; additional copies may be obtained at cost in multiples of 50 .

The Pacific Journal of Mathematics is published quarterly, in March, June, September, and December. The price per volume (4 numbers) is $\$ 12.00$; single issues, $\$ 3.50$. Back numbers are available. Special price to individual faculty members of supporting institutions and to individual members of the American Mathematical Society: $\$ 4.00$ per volume; single issues, $\$ 1.25$.

Subscriptions, orders for back numbers, and changes of address should be sent to Pacific Journal of Mathematics, 2120 Oxford Street, Berkeley 4, California.

Printed at Kokusai Bunken Insatsusha (International Academic Printing Co., Ltd.), No. $6_{4}$ 2-chome, Fujimi-cho, Chiyoda-ku, Tokyo, Japan.

PUBLISHED BY PACIFIC JOURNAL OF MATHEMATICS, A NON-PROFIT CORPORATION

The Supporting Institutions listed above contribute to the cost of publication of this Journăl, but they are not owners or publishers and have no responsibility for its content or policies. 


\section{Pacific Journal of Mathematics}

\section{Vol. 10, No. $3 \quad$ November, 1960}

Glen Earl Baxter, An analytic problem whose solution follows from a simple

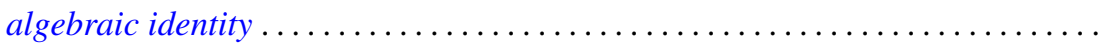

Leonard D. Berkovitz and Melvin Dresher, A multimove infinite game with linear payoff. .

Earl Robert Berkson, Sequel to a paper of A. E. Taylor ......................

Gerald Berman and Robert Jerome Silverman, Embedding of algebraic systems.... 767

Peter Crawley, Lattices whose congruences form a boolean algebra . . . . . ...... 777

Robert E. Edwards, Integral bases in inductive limit spaces . . . . . . . . . . . . . . .

Daniel T. Finkbeiner, II, Irreducible congruence relations on lattices . . . . . . . . . .

William James Firey, Isoperimetric ratios of Reuleaux polygons . . . . . . . . . . . 787

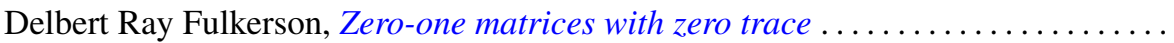

Leon W. Green, A sphere characterization related to Blaschke's conjecture........

Israel (Yitzchak) Nathan Herstein and Erwin Kleinfeld, Lie mappings in

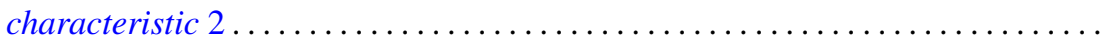

Charles Ray Hobby, A characteristic subgroup of a $p$-group .................

R. K. Juberg, On the Dirichlet problem for certain higher order parabolic

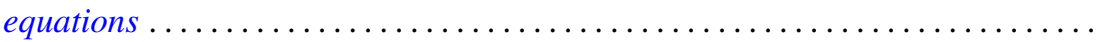

Melvin Katz, Infinitely repeatable games ......................

Emma Lehmer, On Jacobi functions . . . . . . . . . . . . . . . . . . . . . . . . .

D. H. Lehmer, Power character matrices

Henry B. Mann, A refinement of the fundamental theorem on the density of the sum of two sets of integers.

Marvin David Marcus and Roy Westwick, Linear maps on skew symmetric matrices: the invariance of elementary symmetric functions . .

Richard Dean Mayer and Richard Scott Pierce, Boolean algebras with ordered

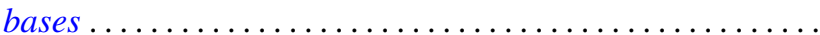

Trevor James McMinn, On the line segments of a convex surface in $E_{3} \ldots$

Frank Albert Raymond, The end point compactification of manifolds ..

Edgar Reich and S. E. Warschawski, On canonical conformal maps of regions of arbitrary connectivity

Marvin Rosenblum, The absolute continuity of Toeplitz's matrices...

Lee Albert Rubel, Maximal means and Tauberian theorems . .

Helmut Heinrich Schaefer, Some spectral properties of positive linear operators

Jeremiah Milton Stark, Minimum problems in the theory of pseudo-conformal transformations and their application to estimation of the curvature of the invariant metric.

Robert Steinberg, The simplicity of certain groups ...

Hisahiro Tamano, On paracompactness. .

Angus E. Taylor, Mittag-Leffler expansions and spectral theory .

Marion Franklin Tinsley, Permanents of cyclic matrices ...... . 